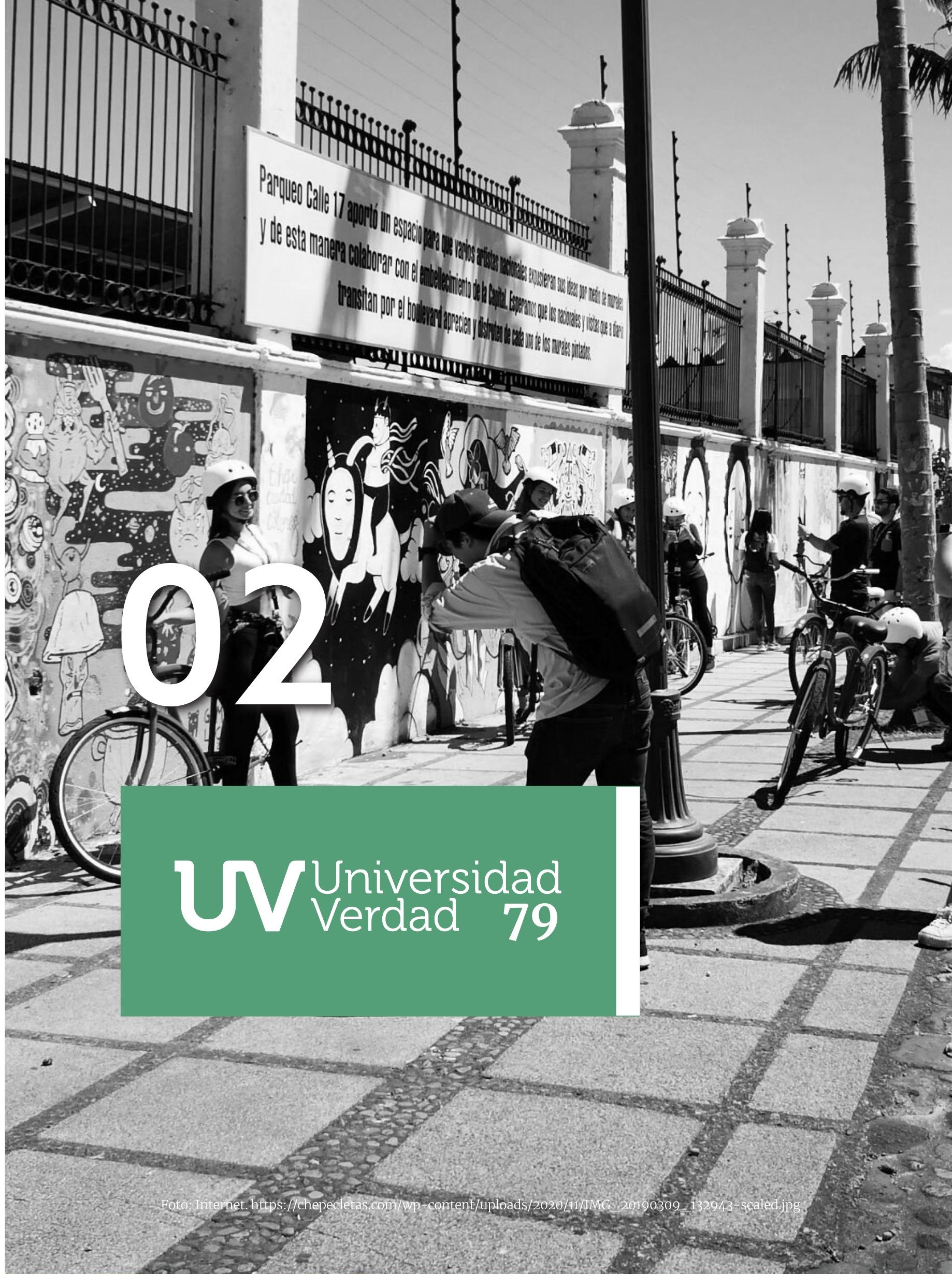




\title{
CHEPECLETAS: UN COLECTIVO A FAVOR DE LA MOVILIDAD URBANA SOSTENIBLE
}

\section{ChepeCletas: A collective in favor of sustainable urban mobility}

\author{
(iD) Laura Parajeles Jiménez, Universidad de Costa Rica (Costa Rica) \\ (laura.parajeles@ucr.ac.cr) (https://orcid.org/0000-0003-0458-2370) \\ (iD) Cristian Silva Jiménez, Universidad de Costa Rica (Costa Rica) \\ (cristian.silva@ucr.ac.cr) (https://orcid.org/00o0-0003-4120-6454)
}

\section{Resumen}

En Costa Rica se encuentra el colectivo ChepeCletas que ha encauzado sus esfuerzos en mejorar la calidad del ambiente, a través del uso de bicicletas y las caminatas, así como la recuperación de espacios para la intervención pública, con el fin de convertir a la capital en un espacio para todas las personas. Por tanto, este artículo realizó un análisis dentro del colectivo, basado en los marcos de referencia de diagnóstico, pronóstico y motivo, dando como resultado mayor conciencia del impacto ambiental que genera el uso excesivo de vehículos privados, la construcción de un grupo que se siente como familia, incrementando el empoderamiento ciudadano para el cambio social, así como el interés por la ciudad y la necesidad de retomar los espacios que siempre fueron públicos; pero habían sido olvidados. Utilizar los marcos de referencia permite comprender, desde la visión de los integrantes, qué es, qué permite y qué se hace en dicho colectivo. Sumado a esto, se debe recalcar que este artículo aporta nuevos conocimientos respecto del tema, visualiza otra forma de hacer política desde abajo y crea espacios que integran cada día a más personas.

\section{Palabras clave}

Movilidad, acciones colectivas, ambiente, política.

Keywords

Mobility, collective actions, environment, politics.

\begin{abstract}
In Costa Rica, where the ChepeCletas collective is to be found, efforts have been channeled to improve the quality of the environment by using bicycles and walking, as well as the recovery of spaces bound for public intervention to turn the capital into a space for all people. Therefore, this article conducts an analysis within the group based on the diagnostic, prognostic and motive reference frameworks which result in a greater awareness of the environmental impact than the excessive use of private vehicles generates. Additionally, the construction of a group that feels like family increasing citizen empowerment for social change, as well as interest in the city and the need to retake spaces that were always public but had been forgotten. The use of reference frames allows us to understand, from the point of view of the members, it's nature, what allows it to be and what is done in said group. In addition to this, it should be emphasized that this article provides new knowledge on the subject, visualizes another way of doing politics from below and creates spaces that integrate more people every day.
\end{abstract}


1.

\section{Introducción}

Los movimientos sociales y las acciones colectivas han permitido crear, visibilizar y retomar iniciativas, ideas, formas de ver el mundo, descontentos, entre otros. Su practicidad los posiciona como una forma de hacer política desde la ciudadanía, para generar cambios en las estructuras sociales, como consecuencia de, en algunas ocasiones, la inacción de los actores y actoras políticas y la falta de atención a las necesidades de los ciudadanos. Siguiendo la idea anterior, se puede complementar con lo que menciona Tarrow (1997), quien afirma que estas "Tienen poder porque desafían a sus oponentes, despiertan solidaridad y cobran significado en el seno de determinados grupos de población, situaciones y culturas políticas" (p. 20), consiguiendo mayores oportunidades para lograr sus objetivos y metas.

Las diferentes acciones colectivas, tanto físicas como virtuales, han logrado posicionarse a través del mundo, y Costa Rica no es la excepción. En la historia del país se han encontrado múltiples movimientos sociales y acciones colectivas con una variedad de repertorios de acción enfocados tanto a quienes toman decisiones como a la ciudadanía misma. Dentro de estos se encuentra ChepeCletas, una iniciativa de Roberto Guz- mán ${ }^{1}$ que busca retomar la movilidad urbana y la apropiación de los espacios de la capital josefina a través de medios de transporte que colaboren con el cuidado del ambiente como las bicicletas y las caminatas.

ChepeCletas va más allá de un movimiento de personas que incentivan el uso de transportes sostenibles, y es la razón fundamental para elaborar este artículo de investigación. En el presente trabajo se podrá encontrar dos puntos importantes: en el primero, se presenta el colectivo, las actividades que ha hecho, así como las iniciativas que apoyan en conjunto con la ciudadanía y otros actores. El segundo presenta un análisis de los marcos de referencia, tanto de su fundador como de quienes forman parte de ChepeCletas.

\section{2.}

\section{Estado de la cuestión}

En esta sección se podrá encontrar, de manera general, un estado del arte sobre la recuperación de espacios urbanos a nivel internacional y nacional como parte de las prácticas de intervención ciudadana para el aprovechamiento de las urbes, a través de acciones colectivas y repertorios de acción. Por tanto, el trabajo de Páramo (2011) es de suma importancia en este punto. El autor plantea que, dado el crecimiento poblacional y la forma en que se desarrolla la vida, se ha dado un decaimiento de la vida social en algunos espacios públicos, por lo que es necesario encontrar prácticas que contribuyan con el mantenimiento de la vida en la ciudad y a la vez mejore la calidad de los ciudadanos. Plantea que es necesario comenzar a integrar lo que él llama "Comportamientos Urbano Responsables" tales como: no fumar en espacios públicos, utilizar transportes alternativos, como la bicicleta y el transporte público, reciclar, ahorrar en recursos hídricos y eléctricos, entre otros.

1. Roberto Guzmán es fundador y actual director de ChepeCletas. Biólogo de carrera que ha dedicado los últimos años a la promoción de la movilidad sostenible y la recuperación de los espacios públicos josefinos a través de ChepeCletas. Para mayor información, favor visitar https://www.linkedin.com/in/robertoguzmanf $/$ ?originalSubdomain $=\mathrm{cr}$ 
Asimismo propone una estrategia para la participación activa de las personas donde se debe contemplar la apropiación/aprendizaje de ciertas reglas: una estrategia comunicacional, una metacontingencia y un diseño urbano. De esta combinación, se espera se produzca un beneficio colectivo, un ambiente más sano y equilibrado, mayor dinamismo en las ciudades, mejor calidad de vida para todas las personas, la identificación con la urbe, por medio de la vinculación con la historia y la sostenibilidad de la vida urbana, por medio del aprovechamiento de espacios.

Asociado a la recuperación de espacios que propone Páramo, se puede mencionar el texto de Benítez (2019), "Los límites del derecho a la ciudad como prisma para entender conflictos urbanos: acción colectiva y lenguaje de derechos en la Ciudad $\mathrm{Au}-$ tónoma de Buenos Aires", donde expone cómo el derecho a la ciudad se ha utilizado como consiga política por organizaciones, acciones colectivas y movimientos sociales de la CABA, que disputan el acceso a la vivienda y al espacio público, sin llegar a crear una vinculación con los demás, que enfrentan problemáticas similares. De esta manera, llevan a cabo acciones por el barrio, la villa o un espacio público específico de forma sectorizada y no visualizan la ciudad como un todo.

Una vez analizados los marcos de referencia (diagnóstico, pronóstico y motivos) el autor llega a la conclusión de que hay un obstáculo para crear una colectividad o un marco de significación conjunta, además de que existen contradicciones en el actuar y el planteo de las reivindicaciones con la idea del derecho a la ciudad. No obstante, estas personas mantienen la lucha por el acceso a la vivienda, al espacio público, la reurbanización y el derecho al hábitat, por lo que Benítez (2019) replantea el concepto de derecho a la ciudad para estos casos específicos y propone entender la "ciudad en un lenguaje de derechos", abriendo la posibilidad de comprender que, para el disfrute de la urbe, es necesario acceder a más derechos políticos y sociales que contribuyan a la satisfacción de las demandas y necesidades.

Siguiendo la línea de las luchas ciudadanas para intervenir y recuperar las ciudades, se pueden mencionar dos casos nacionales. El artículo de Fuentes et al (2015) plasma cómo realizaron un trabajo investigativo y una propuesta de bajo costo para intervenir San José, recuperar ciertos espacios públicos y a la vez el derecho a la ciudad, ya que, como ellos mencionan, se han estado violentando la movilidad y la transformación del espacio público.

A través del colectivo Ciudad Alterna se creó la propuesta "Acción con Poder", la cual integra varias estrategias: 1. "Ciudad amigable", que busca recuperar el derecho a la proximidad y la armonía por medio de la creación de espacios para que todas las personas puedan transitar de manera segura, independientemente del transporte que estén utilizando. 2. "Todos somos indigentes" propone la integración de elementos básicos para hacer de la ciudad un espacio que todas las personas puedan utilizar, ya sea bidones de agua gratis, mesas en ciertas bancas o incluso una especie de casita que puede ser utilizada por personas en condición de calle como refugio. 3. "Iconografía" defiende el derecho a la memoria histórica, la cual debe prevalecer en conjunto con las nuevas modificaciones que se le hacen a la ciudad.

Cada una de las estrategias dio como resultado aspectos que se deben destacar, entre ellos, que la ciudad, y, por ende, el espacio público, es una construcción social, sin embargo, las mismas autoridades policiales dificultan la apropiación y la integración de las personas a las diversas actividades que transforman cada punto de la ciudad. No obstante, al recalcar cuáles derechos se estaban retomando en cada propuesta brindó a los ciudadanos un mensaje de civismo y de lucha.

Concordando con los autores anteriores, Lira Sánchez (2015) también propone que los espacios públicos son una construcción social y por tanto deben estar acompañados de mecanismos que fomenten la participación social, creen formas de resistencia, se permita a las personas alzar la voz y vivir la ciudad, así como fortalecer los lazos comunitarios y de acción social, dado que el derecho a la ciudad es fundamental en una época en la cual se ha preponderado a los vehículos restándole importancia, e incluso casi invisibilizando, al peatón. De esta manera, la gestión comunitaria de los espacios ha permitido la identificación de tres ejemplos claros. El primero, se encuentra en las comunidades de los 
Olivos y los Geranios, donde el Comité Pro Parque, de ambos lugares, se propuso tener acceso a espacios verdes, aptos para todas la comunidad, sin distinción de edades.

El segundo caso, se encuentra en la Comunidad de Las Lomas del Río. En esta zona se buscó recuperar espacios considerados como emblemáticos/simbólicos para la comunidad, por medio de la recreación, la prevención de la violencia y la puesta en escena de muestras artísticas. El último ejemplo se refiere al barrio Sevilla, en Dulce Nombre de Coronado, donde han puesto su esfuerzo en la recuperación de dos parques comunales por medio de la intervención de la misma población de la zona, así como la presión a las autoridades municipales encargadas. Cada una de estas acciones colectivas se refiere a lo que el autor plantea como un mayor empoderamiento y participación comunal en pro de la creación y transformación de las ciudades. (Lira Sánchez, 2015)

A modo de corolario es importante tener en consideración que, como idea principal en todos los trabajos, se encuentra la participación ciudadana como eje fundamental para la apropiación de los espacios, la transformación de estos y para mantener las ciudades vivas, evitando ser zonas de tránsito pasajero en ciertas horas y convirtiéndose en desiertos en la noche. Cada una de las investigaciones descritas destaca a las y los ciudadanos como actores principales y como responsables de la modificación de las conductas que dañan los espacios, la lucha por el derecho a la ciudad y como personas con agencia para incidir en los tomadores de decisiones mediante la presión para obtener espacios verdes y dignos que respondan a las necesidades de cada comunidad o colectivo.

\section{3.}

\section{Contextualización del problema}

Costa Rica ha logrado posicionarse a nivel mundial como un país verde y ecológicamente equilibrado, que respeta la biodiversidad. Sin embargo, en grandes partes de la Gran Área Metropolitana (GAM) se vive lo que popularmente se llama "jungla de concreto", llena de edificios, comercios, hacinamiento, carreteras, acumulación de vehículos y la destrucción de zonas verdes para la creación de estacionamientos u otras infraestructuras, de manera desordenada.

Sumado a lo anterior, la población que transita por el GAM y especialmente por el área capitalina del país encuentra, día a día, un problema vehicular que se traduce en embotellamientos masivos en carreteras y calles que son considerados puntos estratégicos para el transporte, a pesar de los esfuerzos de administraciones pasadas por generar un respiro al país con medidas prohibitivas, como lo es la restricción vehicular por placas, la cual impide circular por el Gran Área Metropolitana (San José, Heredia y Cartago) a los vehículos en ciertos días.

Esta situación se ve reflejada en el Informe del Estado de la Nación sobre "Implicaciones en infraestructura y transporte", el cual menciona a Costa Rica como uno de los países latinoamericanos con una mayor densidad en carreteras, sin embargo su capacidad funcional está colapsada por la imposibilidad de expandirse y por la mala planificación cuando fueron creadas, lo que da como resultado volúmenes masivos de vehículos, un desarrollo urbano y económico que se focaliza en el mismo lugar donde se da la mayor movilización de personas y un sistema de transporte público que compite por espacio entre sí ante la duplicidad de rutas y horarios; lo cual se traduce en un sistema que da prioridad al vehículo privado, generando pérdidas en la calidad de vida y competitividad, mayor contaminación y aumento en la factura petrolera (Loría, 2014).

De esta manera, estas situaciones "[...] no sólo complican la movilidad dentro de la GAM, sino que adicionalmente, inducen al ciudadano a incurrir en una serie de costos que se incrementan con el tiempo y que están relacionados con la congestión vial, los accidentes de tránsito, la salud, la contaminación y el deterioro del ambiente". (Loría, 2014, p.18), así como a la reducción de oportunidades para quienes deciden trasladarse a pie o en otras formas alternativas más saludables y amigables con el ecosistema.

Sumado a lo anterior, a pesar de las múltiples formas en las que el ambiente se ve afectado, el sistema legal de Costa Rica brinda un marco de acción al es- 
tablecer, en el Artículo 50 de la Constitución Política: "Toda persona tiene derecho a un ambiente sano y ecológicamente equilibrado. Por ello está legitimada para denunciar los actos que infrinjan ese derecho y para reclamar la reparación del daño causado." (s.p, 1994), por lo que Chepecletas encuentra un espacio para su accionar y pone en el centro la discusión los transportes saludables con el ambiente así como la apropiación del espacio urbano de Costa Rica, disminuir la huella del carbono, economizar dinero, hacer ejercicio y crear ciudades amigables con el ambiente.

Tomando en consideración la problemática expuesta anteriormente, así como la iniciativa planteada por ChepeCletas, se establece como pregunta de investigación ¿Cómo ha incidido la acción colectiva Chepecletas para generar un cambio en la cultura vial costarricense por medio de los transportes alternativos al vehículo privado?

\section{4.}

\section{Método}

Este artículo científico pretende analizar, como parte de las iniciativas ciudadanas que realizan política desde abajo, el caso de ChepeCletas, desde dos puntos de vista. El primero presentará este colectivo en términos de historia, sus eventos, iniciativas, colaboraciones, así como las intervenciones que ha tenido en aspectos políticos. Desde un segundo punto de vista, se analizarán los marcos de referencia de diagnóstico, pronóstico y motivos, ya que estos son referentes que dividen en unidades más pequeñas la realidad y las acciones del colectivo, con el fin de comprenderlo mejor.

Al tratar de entender la forma en que actúan los colectivos y sus repertorios de acción para cambiar las estructuras sociales, es necesario una base epistemológica que permita vislumbrar el objeto de estudio, los sujetos que participan así como las formas en que ambos interactúan, por lo que el constructivismo figura como un aspecto principal para estudiar "[...] los hechos desvelando los significados que los seres humanos atribuyen a su conducta y al mundo exterior" (della Porta y Keating, 2013, p. 39).
Sumado a lo anterior, como es característico en este tipo de investigaciones, tendrá una metodología y técnicas cualitativas, dado que permiten tener un acercamiento interpretativista y flexible con el objeto de estudio de forma más cercana. En palabras de della Porta y Keating (2013) "[...] resaltan los casos (que pueden ser un individuo, una comunidad u otra colectividad social como entidades complejas (véase della Porta, cap. XI) y subrayan la importancia del contexto" (p. 43). Dado que las realidades de cada objeto/sujeto de investigación son distintas, y en algunas casos determinada por la socialización primaria y secundaria, la investigación cualitativa junto con sus técnicas permite visualizar el fenómeno social y con ello establecer cuál sería su capacidad de agencia a partir de su organización como colectivo social, evitando las generalizaciones y profundizando en su accionar, sus interrelaciones y sentimientos frente al sistema urbano nacional de transportes.

Lo anterior tiene relación directa con la forma en que se estudiará a ChepeCletas, ya que a partir de los marcos de referencia para la acción colectiva (diagnóstico, pronóstico y motivos) se podrá comprender parte de las razones que los hacen pertenecer a dicho grupo, los problemas que enfrentan así como los sentimientos que los unen, ya que son "[...] esquemas interpretativos de la realidad que inspiran y legitiman las actividades y campañas no ya de un individuo sino de un movimiento social" (López Maya, 2002, p. 31).

La base de la investigación será una revisión bibliográfica que podrá triangularse con la información recabada de los participantes de ChepeCletas y del fundador. Lo anterior, con el fin de analizar de manera completa el colectivo, su incidencia dentro y fuera de la capital josefina y establecer como la acción colectiva ha logrado generar espacios de intervención ciudadana y un impacto en la política. Para esta etapa se sistematizaron varios documentos académicos y artículos de periódicos digitales a partir de una base datos de Excel creada en el segundo semestre del 2017.

Se estableció, como carácter de inclusión, tener relación con al menos una de las siguientes particularidades: 1. Ser una acción colectiva, 2. Intervenir el espacio público, 3. Tener en su contenido marcos de referencia de diagnóstico, pronóstico y soluciones, 
4. Mencionar actividades de ChepeCletas y 5. Hacer referencia a la situación país en términos de viabilidad vehicular. La información seleccionada se encuentra mencionada a lo largo del presente artículo y fue recabada a partir de una búsqueda general en la web, así como por medio del Sistema de Bibliotecas, Documentación e información (SIBDI) de la Universidad de Costa Rica, al cual tienen acceso sus estudiantes y funcionarios.

Para la recolección de información, se utilizarán dos técnicas, las cuales están enfocadas en generar interacción social entre los investigadores y los sujetos de investigación. Estas se aplicaron al fundador de ChepeCletas, Roberto Guzmán, y a los integrantes del colectivo, que deseen participar. Para el primero, se escoge la entrevista como el medio principal para obtener información; y para los segundos, el cuestionario. Se escoge la entrevista ya que "se utiliza para conocer opiniones, actitudes, ideas o interpretaciones de la persona entrevistada sobre un evento o fenómeno social que ya pasó"'" (Sibaja, 2013, p.101) Por otro lado, el cuestionario permite obtener respuestas de personas identificadas con ciertos tópicos. Sibaja (2013) lo establece como "El conjunto de preguntas que se utilizan para interrogar a la gente sobre asuntos muy variados [...]" (p.128).

Sumado a lo anterior, se escogen estos instrumentos porque su aplicación tiene mayor flexibilidad, ya que se ajusta al entrevistado, permitiendo aclarar términos y reducir los formalismos. Por tanto, puede adaptarse al caso específico de cada intervención, según la visión de la persona entrevistadora (Díaz et al, 2013)

Respecto de las entrevistas semiestructuradas, se realizaron dos entrevistas de este tipo en dos ocasiones a Roberto Guzmán. La primera se llevó a cabo el día 12 de Setiembre de 2017 por medio de una llamada telefónica para conocer, a partir de una única sección, detalles generales del colectivo, algunas de sus iniciativas y su activismo, sus opiniones sobre la libertad de tránsito y el ambiente ecológicamente equilibrado. Esta primera técnica de recolección de información permitió crear una entrevista más robusta llevada a cabo el 2 de Octubre y crear los primeros esbozos del trabajo en conjunto con los documentos encontrados.
La segunda entrevista fue llevada a cabo de manera personal en la oficina de ChepeCletas, en San José centro. El guion de esta se estructuró en 4 secciones: I. Información general, II. Problemática, III. Soluciones y IV. Motivos. Fue enfocada en recopilar información para crear el análisis de los marcos de referencia, así como detalles generales de sus actividades y la historia del colectivo. Es importante mencionar que ambas fueron transcritas para uso del investigador.

El cuestionario se aplicó a 15 personas que participaron en las diferentes movilizaciones que ha hecho ChepeCletas. El cuestionario realizado es abierto, para que resalte la información que brindarán los sujetos de investigación. Fue aplicado el día 30 de Octubre de 2017, tomando en cuenta aspectos como el tipo de actividades a la que asiste (caminatas o recorridos en bicicleta), la opinión sobre ChepeCletas y su labor como acción colectiva, apreciación del grupo (familia/grupo de acción), motivo para participar, aspectos sobre las carreteras, prioridad al ciclista, transporte alternativo, entre otros.

Para esto cada persona llenó un formulario en línea que la investigadora les facilitó, lo que permitió una sistematización automática de las respuestas dadas. Además, se estructuró de la siguiente manera: I. Parte: Información personal, II. Parte: Participación en las actividades de ChepeCletas, III. Parte: Relación-sentimientos con el colectivo y demás participantes, IV. Parte: Opinión sobre la infraestructura vial costarricense, la apropiación de espacios y el uso de transportes alternativos. 
5.

\section{Resultados}

\subsection{ChepeCletas: una alternativa colectiva al siste- ma tradicional de transportes}

ChepeCletas es una acción colectiva que busca retomar el espacio urbano en San José. Nace en 2010 gracias a Roberto Guzmán, quien tiene como objetivo principal "Convertir a San José en una ciudad más activa, más caminable, más bici-amigable y más humana" (ChepeCletas, s.f). Es decir, mediante la apropiación del espacio público, se busca generar un cambio en la forma en la que se percibe la ciudad y con ello hacer de esta, un sector más dinámico y limpio. Para esto, Roberto se apoya en los recorridos que organiza, ya sea en bicicleta, caminatas o, en casos esporádicos, en bus.

Cada una de las actividades que se realizan en pro de la reapropiación de la capital responden a la necesidad de crear más espacios de intervención pública que fomenten el disfrute de la ciudad, la recreación sana, el desarrollo de los comercios, así como la posibilidad de crear espacios de intervención para todas las edades y gustos. Cada una de las actividades que se realizan tienen un fin explícito, pero a la vez contribuyen a lo que planteaba Henri Lefèbvre: el derecho a la ciudad y la integración de las personas para transformar la ciudad. Si bien este autor planteaba una visión europea enfocada luego de la Segunda Guerra Mundial, su aporte aún permanece vigente. Parte de su ensayo explicado por Molano Camargo (2016) plantea que:

El urbanismo moderno, según Lefebvre, había generado una mayor segregación espacial, el predominio del valor de cambio del espacio ahora mercantilizado, y la imposibilidad de que los trabajadores pudieran participar en las decisiones sobre la ciudad, confinados en una vida urbana enajenada por el consumo, la fragmentación de la cotidianidad y la exclusión espacial. Por ende, Lefebvre enunció el derecho a la ciudad como el retorno de la clase obrera a la ciudad en calidad de productora del espacio y usufructuaria de su valor de uso. La experiencia urbana de la clase obrera y su cotidianidad no enajenada serían la fuente de las nuevas utopías urbanas. Esta estrategia de la revolución urbana socialista planteaba que la lucha obrera debía estar acompañada de iniciativas de investigación urbana (con iniciativas como las que planteaba el mismo Lefebvre) y acción política que posibilitarían a la clase obrera apropiarse de la ciudad, y así habilitarse para incidir en las decisiones sobre la ciudad. (p.6)

Lo expuesto anteriormente es una crítica que se encuentra presente, dado que la falta de intervención económica, social y política han creado en la capital josefina una segregación espacial, dando como resultado espacios opuestos, con tan solo algunas cuadras de distancia. Por una parte, hay lugares de la capital, solitarios e inseguros, marginados y con poco desarrollo, en contraposición a otros, donde existe un comercio activo y se les suele encontrar con muchas personas. Sumado a esto, la reapropiación de los espacios urbanos ha permitido que se rompa con la idea de un San José feo, sucio y abandonado y se haya retomado la idea de disfrutar la capital y sus espacios, dándole la oportunidad a cualquier persona de ser parte de las intervenciones sociales que se han realizado y conocer un poco más la ciudad.

En este marco de acción, Guzmán en conjunto con los integrantes de ChepeCletas tienen claro que es necesario que las personas deben salir cada vez más a espacios de y por recreación para disfrutar lo que ofrece la urbe, el comercio, y quienes organizan actividades para los habitantes del país. Siguiendo esta idea, el fundador de este colectivo plantea que cuando hay muchas personas reunidas, no solo hay una apropiación del espacio sino que se rompe con el discurso que una calle ocupada por policías es un lugar seguro. También, expone que ahí "es donde cree que la institucionalidad tiene una tarea clave: trabajar en conjunto con la gente para transformar el espacio público" (Salazar, 2015).

El derecho a la ciudad y, como se planteaba anteriormente, el retorno de las personas a espacios urbanos se ha catalizado de forma positiva a través de las redes sociales. Esta herramienta ha sido utilizada por Guzmán, quien en el Facebook de ChepeCletas anuncia las actividades que se realizarán como colectivo (Ver figuras 1, 2 y 3). Esto les ha permitido tener ma- 
yor incidencia, conseguir el apoyo de la Municipalidad de San José, así como de otros colectivos urbanos y la difusión que le dan los medios nacionales escritos a las actividades que realizan.

\section{Figura 1}

Página principal en Facebook

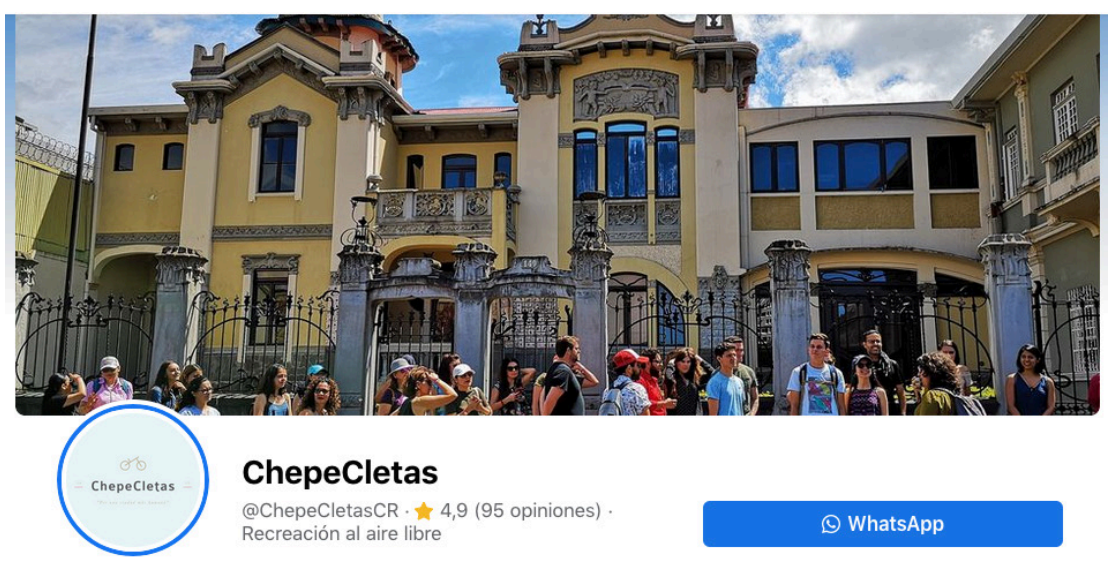

Fuente: Adaptado de página principal de Facebook de ChepeCletas. [Captura], por ChepeCletas, 2021, (https://www.facebook.com/ChepeCletasCR). Obra de Dominio Público.

\section{Figura 2}

Actividad Francia en Costa Rica



Fuente: Adaptado de página principal de Facebook de ChepeCletas. [Captura], por ChepeCletas, 2021, (https://www. facebook.com/events/3082792902005792/?ref=newsfeed). Obra de Dominio Público. 


\section{Figura 3}

Celebración del Día Mundial de la Bicicleta

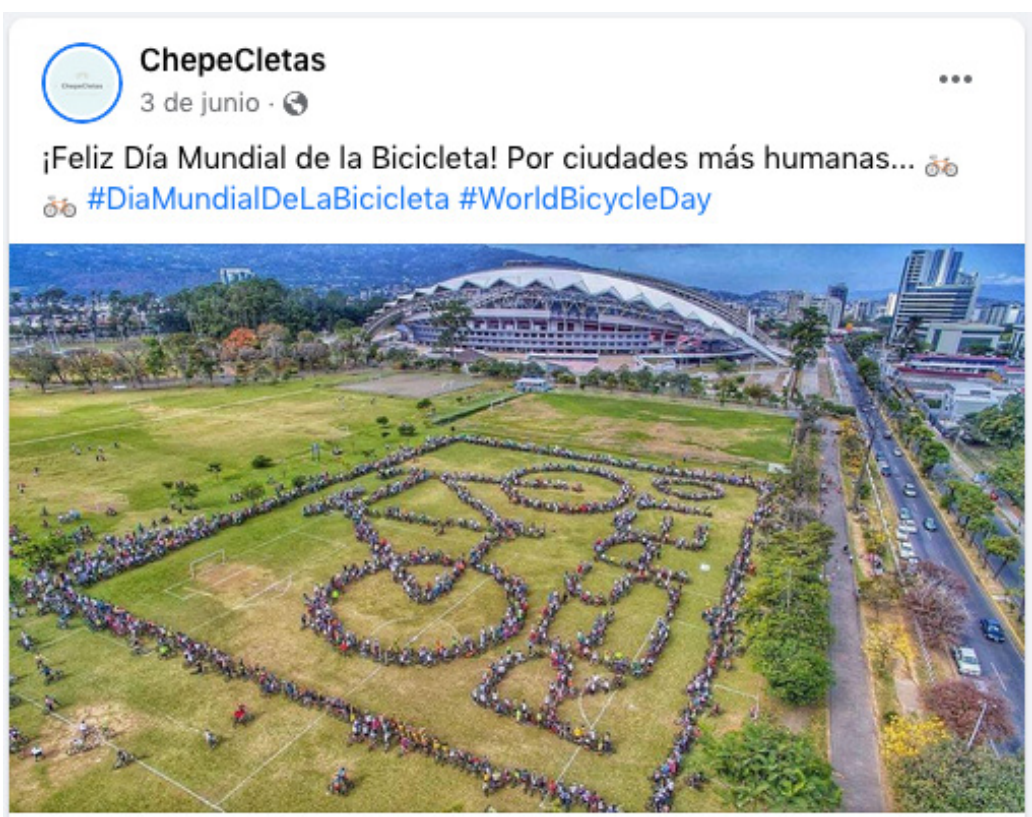

Fuente: Adaptado de página principal de Facebook de ChepeCletas. [Captura], por ChepeCletas, 2021,(https://www. facebook.com/ChepeCletasCR/photos/a.148938455143716/4005825572788299/). Obra de Dominio Público.

Según Roberto Guzmán, esta acción colectiva se presenta como pacifista, pero activa a la hora de movilizar personas cada miércoles y fines de semana, ya sea a pie o en bicicleta. Además, menciona que es necesario redistribuir y equilibrar el espacio público, en donde se le dé prioridad al peatón, luego, a los ciclistas y, por último, al transporte público (Guzmán, comunicación personal, 12 de setiembre de 2017).

Ejemplo de esto es el recorrido realizado para concientizar sobre el cáncer de próstata. "El objetivo es apoyar a la campaña de lucha contra el cáncer de próstata, cuyo mes es noviembre. Por ello, nos pusimos calzoncillos", comentó Roberto Guzmán de ChepeCletas en un comunicado" (La Nación, 2015).

Ibarra (2000) establece que los movimientos sociales son acciones colectivas que reaccionan en función de un conflicto o disconformidad, haciendo de esta acción un proceso visible, o como lo califica el autor, "una llamada de atención" a la sociedad y al Estado. De esta manera, se puede decir que ChepeCletas abarca un tema bien definido: la apropiación del espacio público para lograr una ciudad para todos; sin embargo, mediante sus intervenciones, también colabora con otras causas.

Tomando en consideración lo anterior, el contexto de la ciudad josefina se presenta como el móvil por el cual ChepeCletas decide intervenir. Dicho colectivo comienza con pocos actores, tres específicamente. Sin embargo, con el paso de los años se han llegado a sumar una gran cantidad de personas, haciendo de San José un espacio grupal al que todos se pueden integrar. 
Esta visión va de la mano con el paradigma de la movilización de recursos. Según Gamba (2011) este enfoque prepondera la utilización de los recursos con los que cuenta el colectivo a favor de sus acciones para aprovechar las estructuras de oportunidad política. En el caso de ChepeCletas y otras organizaciones que promueven la cultura en la ciudad, abren el espacio para promover el transporte alternativo. Asimismo, en el contexto actual, donde las pressson continuas, van posicionando la idea de la redistribución del espacio y el libre tránsito.

Retomando a Ibarra (2000), los movimientos sociales adquieren una identidad colectiva, la cual los identifica y, a la vez, diferencia de otros grupos. Esto significa que pueden evolucionar y transformarse para perdurar a lo largo del tiempo, hasta que sus demandas sean escuchadas y cumplidas. Ante esto, la realidad de ChepeCletas no está lejos de lo antes planteado, ya que

Como cuenta Roberto, nace en el 2010 con ese concepto, pero va mutando, evolucionando, mejorando. Quizás con aportes también de Art City Tours, con quienes colaboran, o por ideas propias, pero fueron desarrollando el concepto de devolverle a San José la cultura urbana, esa cultura en la que la gente va sin temor a los parques, calles y rincones de la capital. En su cabeza, los de Chepecletas querían que la gente volviera a poblar "chepecentro", se conociera, compartiera en los espacios comunes y recuperara así el sentimiento de comunidad, de vecindario en la últimamente olvidada capital. (Boncompagni, 2014)

Sumado a lo anterior, la lucha constante que ha tenido Roberto Guzmán y su colectivo ha logrado grandes avances en la institucionalidad de Costa Rica. Prueba de esto son los acuerdos con la Municipalidad de San José y con el Ministerio de Obras Públicas para la realización de más ciclovías. Por otra parte, algunas figuras políticas se han mostrado interesadas en el proyecto y su expansión a otras provincias, como Cartago (Boncompagni, 2014).

De esta manera, su abordaje se presenta como necesario para analizar la relación entre un colectivo urbano y pacífico con la incidencia dentro de los aspectos generales de la ciudadanía, la institucionalidad costarricense y en la forma en la que va cambiando el transporte dentro de la ciudad, en un contexto donde la necesidad de nuevos medios alternativos para llegar al lugar de destino se ha hecho prioridad para las diferentes agendas del país y para la misma ciudadanía.

Relacionado con la realidad nacional y con lo anterior, el colectivo pretende cambiar la percepción negativa que se ha creado sobre San José, ya que es una ciudad activa durante el día y queda totalmente vacía en las noches, haciendo que las personas no asistan por gusto sino por alguna obligación. Sumado a esto, existe la condición de las presas, la inseguridad, la contaminación y un mayor número de autos que le restan movilidad a la ciudad (Guzmán, comunicación personal, 2 de octubre de 2017).

En suma, San José se presta para realizar muchas actividades. Menciona Guzmán (comunicación personal, 2 de octubre de 2017) que hay falta de información de todas las actividades que se realizan. Se debe enseñar que está pasando en la capital y, principalmente, su historia, para que las personas vuelvan a estos espacios y no consideren su estancia como algo terrible.

Respecto a sus acciones se encuentran diferentes rutas dentro de sus recorridos. Con la bicicleta se visitan lugares como Curridabat, Moravia, Desamparados, la Sabana y sus alrededores. Durante las caminatas se tienen como atractivo Amón, los mercados de San José, recorridos de café, de árboles, entre otros. Otra opción que se realiza una vez al mes es el recorrido con la Periférica.

Cabe mencionar como se han ido diversificando los procesos de culturización y recuperación del espacio. En un inicio el proyecto se basaba en bicicletas, ahora se extiende a caminatas y la utilización de transporte público. Pero como una de las metas es integrar bici-taxis, con el fin de ampliar la gama de transporte alternativo, ayudar al ambiente y contribuir con la sociedad y la disminución de presas (Guzmán, comunicación personal, 2 de octubre de 2017).

Según el fundador, ChepeCletas ha generado un alto impacto dentro de la sociedad costarricense, ya que en las actividades han estado participando en pro- 
medio 50 personas de edades muy variadas, durante las caminatas. No hay distinción de edades, ya que llegan desde jóvenes hasta adultos mayores, en donde la combinación hace que todos se sientan cómodos dentro del grupo. En las actividades con bicicletas llegan en promedio los miércoles 50 personas de personas mayores de 30 años; por otra parte, los fines de semana llegan más de 100 al ser una actividad más familiar (Guzmán, comunicación personal, 2 de octubre de 2017).

Agregando a lo anterior, a pesar de que ChepeCletas es una acción colectiva que se desarrolla en San José, han logrado extenderse con actividades en bicicleta a otras regiones del país. Esto no es de forma permanente como se vive en la capital, pero contribuye a la concientización y a integrar a nuevas personas dentro de la realidad del colectivo. Según menciona en la entrevista Guzmán (comunicación personal, 2 de octubre de 2017) han estado ubicados en Alajuela, la Unión de Tres Ríos, Cartago, Escazú, Belén, Nicoya, entre otros. Bajo este aspecto, la idea principal siempre se mantiene en las calles josefinas, pero están anuentes a compartir la logística con otras regiones para seguir creciendo día a día.

A futuro, según informa Guzmán (comunicación personal, 2 de octubre de 2017), se pretende seguir posicionando el colectivo mediante una frecuencia alta de actividades con el fin de llegar cada día a más personas. Menciona, además, el deseo de crear contenido propio para no replicar el contenido de terceros y seguir desarrollando retretas en espacios públicos para seguir atrayendo a las personas a la capital y que estas disfruten y convivan en el espacio.

Otros espacios de impacto relacionado con la realidad nacional es continuar con el convenio que tienen con el Mercado Borbón, ayudar a las Universidades públicas con los proyectos de voluntariado, conseguir que las empresas privadas busquen actividades que se desarrollan en el centro y no tanto en espacios alejados (Guzmán, comunicación personal, 2 de octubre de 2017) Es decir, se ha comenzado a trabajar con poblaciones pequeñas para ir, poco a poco, recuperando el espacio público que le pertenece a la ciudadanía y no solo a los automotores privados, ya que la calle y las carreteras son espacios públicos creados para el disfrute de las personas.
Por lo tanto, a modo de resumen de este apartado, la razón de ser de estos espacios de intervención viene de la mano con la búsqueda de un futuro más eco amigable con el ambiente, la liberación de las calles, la creación de medios alternativos que sean eficientes, la recuperación del espacio urbano para darle una nueva cara a las calles josefinas. Sin duda alguna, dentro de un solo colectivo buscan abarcar muchos aspectos que están interrelacionados unos con otros, generando una cadena de eslabones que, día a día, se hace más fuerte y hace posible visualizar un San José distinto.

\subsection{Marcos de referencia de ChepeCletas}

La acción colectiva ChepeCletas pone en el centro de la discusión los transportes saludables con el ambiente como lo son las bicicletas y las caminatas así como alternativos a los vehículos privados con el fin de la apropiación del espacio urbano de Costa Rica, disminuir la huella del carbono, economizar dinero, hacer ejercicio y crear ciudades amigables para todas aquellas personas que pasan por estos lugares de intervención, lo cual los convierte en una acción colectiva que busca generar una incidencia social y política. Cada una de sus intervenciones es una forma de demostrar la agencia que han logrado adquirir con el paso del tiempo y sentar un antecedente para demostrar que el derecho a la ciudad es fundamental para las personas.

En este punto es importante retomar la información recabada durante las entrevistas y el cuestionario realizado, así como la idea de los marcos, los cuales son utilizados para comprender mejor una realidad y movilizar a cierta cantidad de personas interesadas en un tema específico o en cierta temática con el fin de llevar una acción a cabo para cambiar una determinada situación. Chihu (2000) citando a Snow y Berford propone 3 marcos que segmentan la acción colectiva en varios aspectos, con el fin de comprender mejor su accionar: diagnóstico, pronóstico y motivos.

El primero, se refiere a la identificación de un problema, por qué es problema, los responsables o quién tiene la culpa. El segundo, hace referencia a las posibles soluciones y lo que se debe hacer para darle respuesta al problema. Este incluye estrategias, tác- 
ticas, presión y negociación. El último marco menciona las razones y motivaciones que mueven a las personas, muchas veces estas responden al carácter subjetivo y emocional de la situación y no de forma racional.

\subsection{Diagnóstico}

Guzmán (comunicación personal, 2 de octubre de 2017) plantea que se le ha cedido completamente las calles a los automóviles, como problema principal. Se ha generado un desequilibrio, en donde casi el 90\% de los espacios urbanos está ocupado por carros, sin embargo, si alguien desea cambiar esto se ve como algo imposible de realizar. Las personas reclaman cuando hay iniciativas para darle prioridad al peatón, lo cual ha generado un gran costo político cuando se realizan acciones a favor del tema. Es decir, estos proyectos no son populares, lo que provoca que se pierdan votos en las elecciones, pero hay otros sectores que ven beneficiosos estos avances.

También expone que algunos de los responsables son las mismas personas por insistir en el uso de transporte privado/propio y las municipalidades y el Estado, ya que no están creando soluciones para evitar las presas dentro de las calles josefinas. Las personas están comprando hasta dos carros, con el fin de evitar la restricción vehicular, utilizando diferentes placas para poder entrar a San José. Además, buscan rutas alternas en vez de utilizar los medios de transportes alternativos. Destaca, a la vez, que algunos de los responsables indirectos del caos vial que se genera en las calles son los bancos y las empresas vendedoras de autos. Estas desean vender cada vez más y brindan las facilidades, pero no analizan que también tienen una cuota de responsabilidad por la contaminación, los accidentes y la congestión en las calles. Es importante cambiar esta cultura, en donde no se prepondere la utilización del carro (Guzmán, comunicación personal, 2 de octubre de 2017).

Un problema intrínseco dentro de esta problemática es el transporte al ser parte contaminante número uno. Sobre esto, el director de ChepeCletas no cree que los vehículos eléctricos sean la solución, ya que se continúa con la presencia exagerada de automó- viles, aumentando la presa y la emisión de gases, ya que no todas las personas tienen la posibilidad de adquirir este tipo de autos (Guzmán, comunicación personal, 2 de octubre de 2017).

Sumado a esto, los participantes de este colectivo destacan que uno de los problemas principales es el estado de las calles. La mayor parte de las respuestas se basan en que estas están "malas", "muy malas" o "regulares" lo que afecta a la circulación. Además, Andrea (comunicación personal, 30 de octubre de 2017) puntualiza que las personas, a pie y en bicicletas, corren más peligro en la calle, siendo este uno de los problemas principales a los que se enfrenta esta población. De esta manera establece "[...] son los eslabones más indefensos porque hay más personas en buses, peatones y bicis [...]" Esta visión también la comparte Nuria (comunicación personal, 30 de octubre de 2017), quien también los considera indefensos ante los vehículos

Por otra parte, Andrea (comunicación personal, 30 de octubre de 2017) menciona que "[...] los carros ocupan más espacio", lo cual es complementado por José (comunicación personal, 30 de octubre de 2017) quien aborda este tema mencionando que implementar más transporte público no cambiará la situación de caos vial en las calles josefinas, dado que "La verdad x más transportes que ponga seguirá las presas" Nuevamente Nuria (comunicación personal, 30 de octubre de 2015), de forma acertada, expone que "Las calles están colapsadas" como consecuencia del gran uso de vehículos.

De esta manera, se puede decir que los integrantes de ChepeCletas creen que el problema principal se encuentra en las calles. Estrictamente en el desorden que se presenta por la gran cantidad de carros que entran y salen de la ciudad, sumado de la inseguridad para aquellos que viajan en bicicletas o deciden caminar determinados trayectos.

\subsection{Soluciones}

Sobre este tema, Guzmán (comunicación personal, 2 de octubre de 2017) menciona que gira en torno a dos grandes iniciativas: invertir en transporte público haciéndolo más eficiente y dejar de utilizar el 
carro. Destaca que en ciertas ocasiones la creación de más espacio dentro de las carreteras no hará efecto alguno si las personas continúan comprando más carros. Por el contrario, es necesario promover el uso de autobuses, los cuales logran movilizar a más de 50 personas, y en un carro viajan, actualmente, 1.6 personas. De esta manera no se debe incentivar el uso de vehículos privados de ningún tipo, ya sean eléctricos, de gasolina, hidrógeno.

Expone que es necesario crear un sistema de transporte público que no involucre solo rutas. Esto se refiere a interconectar las rutas y troncalizarlas, de manera que solo un bus entre a la ciudad de San José, evitando más carros dentro de las calles josefinas. Relacionado con lo anterior, se deben implementar planes piloto. Guzmán (comunicación personal, 2 de octubre de 2017) enfatiza que "Estos pueden fallar, pero de ahí se aprende para innovar"

Por otro lado, menciona otras soluciones que deben estar dentro de la agenda de los partidos políticos. Entre ellas, buscar la manera en la que las personas se sientan más cómodas caminando, crear espacios bajo el estándar de accesibilidad universal, mayor iluminación, seguridad, arbolización, cobro por congestionamiento, limpiar las calles de los automóviles que estén mal parqueados, darle más facilidades a quienes van caminando por las calles y no a quienes necesitan estacionar. De esta manera, se debe pensar primero en la persona y luego en el carro (Guzmán, comunicación personal, 2 de octubre de 2017)

Asimismo, menciona que se debe presionar a algunos actores del gobierno y por otra parte se deben aliar con algunos sectores afines para tener más presencia e impacto. En este caso, los diputados son figuras claves para lograrlo, así como los diferentes Ministerios. Actualmente el colectivo está involucrado con el Ministerio de Educación Pública, Ministerio de Obras Públicas y Transportes, Ministerio de Seguridad, han apoyado a la Asamblea Legislativa en temas de viabilidad en algunos proyectos de Ley, han trabajado con las municipalidades, Árboles Mágicos, Embajadas, ONU, UNICEF, UNESCO, Universidades, ACNUR, entre otros.

En cuanto a las soluciones o estrategias que tienen los participantes de ChepeCletas para lograr un em- poderamiento de los ciudadanos, y con esto la apropiación de los espacios públicos y la recuperación de la ciudad josefina, primero exponen, con una saturación de respuestas, se debe darle prioridad al peatón, medios de transporte alternativo como bicicletas y la mejora de los transportes públicos. De esta manera, se pretende reducir el espacio que utilizan los automóviles para que la movilización sea más segura.

Tony (comunicación personal, 30 de octubre de 2017) menciona, haciendo referencia a la bicicleta, que "Son medios de transporte que ayudan a reducir la contaminación y además son las personas las que deben disfrutar los espacios urbanos, no lo vehículos ni el smoke" concordando, Daniel (comunicación personal, 30 de octubre de 2017) expresa "[...] en ciudades hechas para carros han olvidado que las personas necesitamos más el espacio que automotores estacionados haciendo presas o contaminando. Un carro ocupa demasiado espacio muerto y más riesgoso para las personas [...]"

Por otro lado, las personas activas del colectivo preponderan la modernización como algo necesario para el desarrollo de Costa Rica, como la colocación de nuevos medios para llegar de un punto a otro. Una gran parte de las respuestas obedecen a la creación de ciclovías, alquileres de bicicletas, mejoras en el transporte público y la innovación con un metro y una gran terminal. Lo anterior se presenta como la solución a la congestión vial, la gran contaminación que se hace por las emisiones de $\mathrm{CO} 2$ y la falta de espacios para convivir.

Luis (comunicación personal, 30 de octubre de 2017) y Nm (comunicación personal, 30 de octubre de 2017) justifican lo anterior. El primero basado en su percepción, donde "[...] es más funcional la movilidad de este modo" La segunda alude a la sociabilidad de las personas. De esta manera, eliminando una parte de los vehículos privados se podrá lograr que "[...] la gente camine más, y se relacione con otra gente."

Por otra parte, las intenciones de cuidar el ambiente no quedan en el olvido. Estos medios alternativos y la reducción de automotores es una de las principales consignas con las cuales se encuentran identificados los participantes de ChepeCletas. El colectivo tiene como estrategia la promoción del 
uso de bicicletas, ya que "[...] es una forma de descongestionar las calles" (Raúl, comunicación personal, 30 de octubre de 2017), "[...] somos transporte limpio [...]" (Joshep, comunicación personal, 30 de octubre de 2017) además de que permite "[...] mayor fluido de las calles y menos contaminación" (Manuel, comunicación personal, 30 de octubre de 2017).

Por último, y no menos importante, la educación forma parte de las soluciones que se entablan. Esta va de la mano con la educación vial, donde los conductores de los vehículos deben aprender a respetar y darle espacio a los peatones y ciclistas. Joshep (comunicación personal, 30 de octubre de 2017) recalca "[...] una mayor educación al conductor automotor de que un ciclista es una vida, no un estorbo, concientizar a la población de automóviles que tanto el peatón como los ciclistas son seres humanos, una vida, son familia de alguien".

Por tanto, el colectivo se encuentra preocupado por la generación de conciencia en todos los sectores, no solo para recuperar el espacio público sino también para cuidar del ambiente, involucrando e invitando a la sociedad josefina a ser parte cada vez más del uso de medios alternativos o no usar el carro. Esto tiene como fin, tanto la reducción de emisiones, mayor fluidez en las carreteras y el cuidado mutuo entre las personas que transitan por la ciudad.

\subsection{Motivos}

Guzmán (comunicación personal, 2 de octubre de 2017) propone que el vínculo que une a las personas a ChepeCletas son los sentimientos. Va desde el sentido de pertenencia, crear comunidad, sentirse acogidos, bienvenidos. Entre las mismas personas se crea un ambiente familiar en donde se puede compartir sin importar la edad. Sumado a esto, ChepeCletas es una acción colectiva que, a pesar de tener sus metas y objetivos identificados, se posiciona y solidariza con otras causas, tales como la donación de órganos, cáncer de mama, Isla del Coco, refugiados, niñez, maltrato animal, cáncer de próstata, movember, muertes en carreteras, entre otros. Ante esto, el fundador expresa que más que acción colectiva son activadores o co- nectores sociales, los cuales desde el 2010 se mantienen movilizando a las personas con todo tipo de causas (Guzmán, comunicación personal, 2 de octubre de 2017)

Los motivos que reúnen a las personas en las diferentes actividades que realiza ChepeCletas son diversos. De esta manera, la mitad de las respuestas mencionan que el colectivo es como una familia, y la otra mitad, como un grupo con el cual realizar actividades en la ciudad. Los motivos que reúnen a todas las personas son variados, pero la mayoría concuerda con Nuria (comunicación personal, 30 de octubre de 2017) es "un grupo que organiza cleteadas por la ciudad antes motivos de relevancia".

Respecto a la solidaridad que tiene ChepeCletas con otras causas, como las que se mencionaron anteriormente, hubo una saturación de respuestas que ven estas actividades con buenos ojos, calificándolas como muy buenas, ya que ayudan a generar conciencia en la población costarricense. Según lo ve Andrea (comunicación personal, 30 de octubre de 2017) se crea "Conciencia Social". Específicamente sobre la multicausalidad. Mario menciona que sí si se ha incidido en la sociedad costarricense "[...] creando conciencia en varios temas como inclusividad, programas de salud como del cáncer de mama entre otras"

Sobre los motivos específicos que han hecho que las personas se hayan sumado al colectivo prepondera la recreación, hacer ejercicio, amistad, compartir y conocer nuevas personas y, principalmente, crear ciudad. No obstante, también existe otro tipo de motivos que deben ser tomados en cuenta, entre ellos "Conocer ciudades en bicicleta [...]" (Mario, comunicación personal, 30 de octubre de 2017), "[...] hacer conciencia sobre el respeto en carretera" (Raúl, comunicación personal, 30 de octubre de 2017), "[...] recorrer los lugares poco visitados en ocasiones o los muy visitados, pero con atención preferencial" (Joshep, comunicación personal, 30 de octubre de 2017), "Son un espacio que entrelaza el deporte-recreación-amigos-cultura y a la vez es un aporte para disminuir el congestionamiento vial con alternativas sostenibles de transporte" (Tony, comunicación personal, 30 de octubre de 2017) y "Son un gran grupo y me encanta salir con ellos" (Cristina, comunicación personal, 30 de octubre de 2017) 
Bajo este esquema, es imprescindible destacar cómo todos comparten algo en común, el cariño que le han tomado a la acción colectiva, independientemente del momento o las razones por las que se hayan sumado. La pertenencia es uno de los cimientos más importantes para que se mantenga fuerte y crezca día a día. Por su parte, Roberto, se ha encargado, por años, de recibirlos y hacerlos sentir parte de un cambio en la ciudad y en la conciencia de las personas. Joshep (comunicación personal, 30 de octubre de 2017) tiene claro esto y menciona que parte de lo que inspira a las personas a continuar participando es "El carisma del líder (Roberto), su forma de resolver situaciones, la manera de entablar amistad, él es el pilar del grupo", por lo cual es un colectivo que se mantendrá vigente y accionando desde abajo en pro de un cambio social sustancial que impacte en las agendas ciudadanas, políticas, de medios de comunicación y en la vida misma en cuanto a la forma de movilizarse.

\section{6.}

\section{Discusión}

Tomando en consideración la información de las entrevistas, el cuestionario y la recopilación de información expuesta anteriormente, se puede decir que los hallazgos sí son los esperados. ChepeCletas es un acción colectiva que ha sabido aprovechar los recursos y el carisma de su fundador para atraer a cada vez más personas. Es una iniciativa que, al igual que muestran los trabajos expuestos en el Estado del Arte, busca reactivar las zonas de la urbe por medio de la participación ciudadana. Además, se reconoce que se ha perdido el derecho a la ciudad dado el modelo de desarrollo que han implementado los países, dándole prioridad a los automóviles y dejando de lado a quienes se transportan en alternativas más eco amigables, como las bicis, a pie o transporte público.

Dicho lo anterior, es necesaria una mayor incidencia ciudadana que exponga las necesidades, para que estas sean colocadas en las agendas políticas y comunicativas, lo que dará como resultado acciones efec- tivas en pro de eliminar los problemas que afectan a una población. Este proceso lo han sabido manejar muy bien dentro del colectivo y sus esfuerzos no son en vano, han dado frutos a través de alianzas y la solidaridad con otras causas. Por lo tanto, existe un avance en el reconocimiento de estos grupos como agentes de cambio y catalizadores de demandas ciudadanas que son expuestas en diferentes puestas en escena.

Lo anterior permite dar respuesta a la pregunta de investigación y establecer que, a partir de las actividades que han realizado, han visibilizado el uso de los medios alternativos como una solución a los embotellamientos que se hacen en las diferentes partes de la GAM, y especialmente en la capital josefina. Aunado a lo anterior, han llegado a entablar fuertes lazos y vinculaciones con la institucionalización pública y privada, afianzando su legitimidad como actores que conocen del tema y han puesto en evidencia la necesidad de un cambio de paradigma en cuanto a movilidad urbana, dado que el modelo implementado desde años atrás ha fracasado por la ausencia de un ordenamiento territorial.

A la vez, ChepeCletas sienta un precedente, demostrando que el derecho a la ciudad es importante para la recuperación de espacios, en este caso, a través de tours por lugares conocidos y poco conocidos, así como el apoyo a comercios en eventos como el Art City Tours, lo que da como resultado la creación de comunidad, espacios seguros, disfrute, la promoción la salud a través del ejercicio, la reactivación económica, la creación de conciencia sobre un ambiente sano, la importancia que tienen las personas en carretera y la existencia de la problemática del costo político que implica para algunas personas apoyar iniciativas de este tipo.

Este estudio reconoce la existencia de marcos de referencia, los cuales permiten tener un conocimiento más profundo y colectivo de las razones por las que están ahí, las problemáticas que enfrentan, así como los sentimientos que les unen. Comprender cada uno de los repertorios de acción que han llevado a cabo y su incidencia dentro de la política cantonal josefina, así como en medios de comunicación, se traduce en una ciudadanía activa y empoderada que ha tomado la decisión de recuperar los espa- 
cios que inicialmente fueron siempre de la sociedad misma y que por el aumento masivo de automóviles se perdieron.

Desde un punto más teórico, se desea traer a colación el artículo presentado por Benítez (2019) en "Los límites del derecho a la ciudad como prisma para entender conflictos urbanos: acción colectiva y lenguaje de derechos en la Ciudad Autónoma de Buenos Aires", el cual se encuentra en el Estado de la Cuestión, y con ello generar una discusión basada en una comparación. De esta manera, ChepeCletas sí llega a crear vinculaciones con otras colectividades, incrementando las posibilidades de llevar sus ideas y repertorios de acción a nuevos espacios, y creando un marco de significación colectiva que genere una ruptura con la idea de las ciudades como zonas de tránsito.

La solidaridad y la identificación con otras causas ha permitido que se dé la lucha por el derecho a la ciudad y a la vez lo que expone Benítez como "la ciudad en un lenguaje de derechos", al existir la idea concreta de la recuperación de espacios, el disfrute de la ciudad y la visualización de causas y luchas por los derechos sociales y ambientales.

Por último, como una nueva línea de investigación, se sugiere analizar ChepeCletas a partir de un enfoque de comunicación digital ${ }^{2}$, al tomar en cuenta sus redes sociales e incluso las acciones que han realizado desde la virtualidad y la importancia de estos mecanismos para producir y reproducir contenidos. En este sentido, este enfoque busca incidir desde el mundo virtual en la realidad como se visualiza en esta investigación.

\footnotetext{
2. Revisar la teoría de la Mediápolis de Roger Silverstone, en su
} libro llamado "La moral de los medios de comunicación"

\section{7.}

\section{Conclusiones}

ChepeCletas nace como iniciativa de Roberto Guzmán, con el fin de crear conciencia sobre la contaminación ambiental que produce el uso excesivo de vehículos. El proyecto se ha enfocado en la ciudad de San José, pero ha logrado extenderse a otras regiones. Como idea principal se utilizan los recorridos en bicicleta y la caminata, con el fin de promover el transporte alternativo. Esta iniciativa ha generado gran incidencia, llegando a participar gran cantidad de personas que se identifican con la causa principal y las demás con las que solidarizan.

Los participantes muestran un gran apego y especial cariño a Roberto, destacándolo como el líder y pilar de la acción colectiva. Bajo este aspecto, se encuentran los marcos, donde el diagnóstico se refiere a la gran contaminación, caos vial, irrespeto a los peatones y ciclistas en las carreteras, la falta de innovación en el transporte público y el costo político que representa fomentar y apoyar iniciativas a favor de un cambio en el paradigma de la movilidad urbana.

En cuanto al pronóstico se establece la recuperación de los espacios públicos por medio de la intervención social y el empoderamiento de los ciudadanos para que exijan y participen de las actividades que se llevan a cabo en la capital. Por último, los motivos están interconectados con la familia y los vínculos de amistad que se han llegado a formar a lo largo del tiempo.

ChepeCletas es merecedor de especial atención, ya que inició con pocos recursos y pocas personas, pero a lo largo de los años ha ido creciendo de manera exponencial gracias a las redes sociales, medios de comunicación y publicaciones en los medios digitales escritos, que informan de las actividades recreativas y artísticas que realizarán o en las que tendrán alguna participación. En este sentido, a partir de esta divulgación, las personas pueden comprender con mayor precisión los pilares en los que se basa el movimiento, como lo son la apropiación de los espacios urbanos, el uso de transporte saludable, el derecho al 
disfrute de la ciudad y la necesidad de intervenir en la creación de espacios más seguros para la misma población costarricense y extranjera.

\section{Referencias}

Benítez, J. (2019). Los límites del derecho a la ciudad como prisma para entender conflictos urbanos: acción colectiva y lenguaje de derechos en la Ciudad Autónoma de Buenos Aires. Población y Sociedad. 26 (1), pp.6-31. https://cerac.unlpam. edu.ar/index.php/pys/article/view/3417/3864

Boncompagni, G. (25 de Octubre de 2014). Chepecletas: con bicicletas y caminatas, devolviendo la cultura urbana a San José. CRhoy. http://www.crhoy.com/archivo/chepecletascon-bicicletas-y-caminatas-devolviendo-lacultura-urbana-a-san-jose/nacionales/

Constitución Política de Costa Rica. [Const] Artículo 50. 10 de junio de 1994. (Costa Rica)

ChepeCletas. [ChepeCletas]. (s.f.). Información. Facebook. https://www.facebook.com/pg/ ChepeCletasCR/about/?ref=page_internal

Chihu, A. (2000). El análisis cultural de los movimientos sociales. La fundación de los marcos. Sociológica.15(42), pp.209-230. https:// www.redalyc.org/pdf/3050/305026734005.pdf

della Porta, D y Keating, M. (2013). ¿CUÁNTOS ENFOQUES HAY EN CIENCIAS SOCIALES? Introducción epistemológica. En D. Porta y M. Keating (Coord.), Enfoques y metodologías de las Ciencias Sociales. Una perspectiva pluralista. (pp. 31-51). AKAI.

Díaz, L; Torruco, U; Martínez, M. y Varela, M. (2013). La entrevista, recurso flexible y dinámico. Investigación en Educación Médica. 2(7), pp. 162-167. https://www.redalyc.org/ pdf/3497/349733228009.pdf
Fuentes, P., Gutiérrez, L., y Porras, M. (2015). intervenciones urbanas por el derecho a la ciudad. revistarquis. 4(1), pp. 29-32. https:// www.revistas.ucr.ac.cr/index.php/revistarquis/ article/view/19978/20175

Gamba, A. (6-8 de setiembre de 2011). Enfoques latinoamericanos en el estudio de los Movimientos Sociales (1989-2003). [Ponencia]. XXVIII Congreso Internacional de ALAS, Distrito Federal, México. https://es.scribd. com/document/218036540/EnfoquesLatinoamericanos-en-El-Estudio-de-LosMovimientos-Sociales-Ponencia-Alas-libre.

Ibarra, P. (2000). ¿Qué son los movimientos sociales? En E. Grau y P. Ibarra (Coord.), Una mirada sobre la red: anuario movimientos sociales. (pp. 9-26) Icaria Editorial.

La Nación. (2 de Noviembre de 2015). Cletear en calzoncillos para concientizar sobre el cáncer de próstata. La Nación. http://www.nacion. com/vivir/bienestar/Cletear-calzoncillosconcientizar-cancer-prostata_0_1521847820. html

Lira Sánchez, D. (2015) Reflexiones sobre los procesos de recuperación de los espacios públicos por medio de la participación activa: De la teoría a la práctica. REVISTARQUIS. 8(1), pp. 72-84. https://revistas.ucr.ac.cr/index.php/ revistarquis/article/download/35799/36670/

López Maya, M. (2002). Protesta y cultura en Venezuela: los marcos de acción colectiva en 1999. CLACSO.

Loría, L. (2014). Implicaciones en infraestructura $y$ transporte. [Ponencia]. VIGESIMOPRIMER INFORME ESTADO DE LA NACIÓN EN DESARROLLO HUMANO SOSTENIBLE (2014), San José, Costa Rica, https:// www.lanamme.ucr.ac.cr/repositorio/ bitstream/handle/50625112500/647/ Loria Infraestructura y movilidad. pdf? sequence $=1 \&$ isAllowed $=y$ 


\section{ChepeCletas:
Un colectivo a favor de la movilidad urbana sostenible}

Molano Camargo, F. (2016). El derecho a la ciudad: de Henri Lefebvre a los análisis sobre la ciudad capitalista contemporánea. Revista Folios. (44), pp. 3-19. https://www.redalyc.org/ pdf/3459/345945922001.pdf

Páramo, P. (2011). La recuperación del espacio público para la formación del ciudadano en comportamientos urbanos responsables. Educación y ciudad. (21), pp. 155-166. https:// dialnet.unirioja.es/descarga/articulo/5704959. pdf

Salazar, D. (8 de Setiembre del 2015). Ciudadanos proponen apropiarse de la ciudad para reinventarla. Semanario Universidad. https:// semanariouniversidad.com/cultura/ ciudadanos-proponen-apropiarse-de-laciudad-para-reinventarla/.

Sibaja, G. (2013). La entrevista: cuestionario abierto, historia de vida y fotografía. En Técnicas cualitativas de investigación (1 Ed, pp. 99). Editorial UCR.

Tarrow, S. (1997). El poder en movimiento. Los movimientos sociales, la acción colectiva y la política. Alianza Editorial. 
